\title{
Correction to: On the use of the outcome variable "small for gestational age" when gestational age is a potential mediator: a maternal asthma perspective
}

Geneviève Lefebvre $^{1,2^{*}}$ and Mariia Samoilenko ${ }^{1}$

\section{Correction}

Following publication of the original article [1], the authors reported that the following four references in Table 2 were incorrect:

Lao et al. [15]

Braken et al. [23] ${ }^{\mathrm{b}}$

Bakhireva et al. [26] ${ }^{\mathrm{b}}$

Clark et al. [28] ${ }^{\mathrm{b}}$

These references have already been introduced in Table 1 and should read:

Lao et al. [30]

Bracken et al. [38]

Bakhireva et al. [41]

Clark et al. [43]

The original article has been updated.

Received: 18 January 2018 Accepted: 18 January 2018

Published online: 29 January 2018

\section{Reference}

1. Lefebvre G, Samoilenko M. On the use of the outcome variable "small for gestational age" when gestational age is a potential mediator: a maternal asthma perspective. BMC Med Res Methodol. 2017;17:165. https://doi.org/ 10.1186/s12874-017-0444-z.

\footnotetext{
* Correspondence: lefebvre.gen@uqam.ca

'Department of Mathematics, Université du Québec à Montréal, C.P. 8888,

Succursale Centre-ville, Montréal, Québec H3C 3P8, Canada

${ }^{2}$ Faculty of Pharmacy, Université de Montréal, Montréal, Canada
} 